\title{
Effects of Overdose of High-Dose Thiamine Treatment
}

\author{
Antonio Costantini ${ }^{1 *}$ and Roberto Fancellu ${ }^{2}$ \\ ${ }^{1}$ Department of Neurological Rehabilitation, School of Physiotherapy, Italy \\ ${ }^{2}$ Neurology Unit, Italy \\ *Corresponding author: Antonio Costantini, Department of Neurological Rehabilitation, Italy
}

Submission: 監 December 03, 2018; Published: 悳 December 06, 2018

\section{Introduction}

We treat Parkinson's disease with high dose thiamine in addition to the classic therapy since 2012. On this topic, we published three studies carried out with Italian patients [1-3]. The treatment consisted in performing 2-3 intramuscular injections of $100 \mathrm{mg}$ of thiamine per week. The highest dosage was reserved to patients whose disease severity was the greater, long past onset, and patients whose weight was more than $90 \mathrm{~kg}$. We also treated patients on anticoagulants. In this case, the thiamine was administrated orally (tablets). We noticed that in order to obtain comparable results of the intramuscular therapy with the oral therapy we needed to administrate a dose of thiamine 140 times when treated with tablets or capsules (personal data, not published). In other words, in order to obtain the same clinical effect of 1 intramuscular injection of $100 \mathrm{mg}$ of thiamine once a week, it was necessary to administrate 14 grams of thiamine orally per week, or 2 grams per day. This dosage, when appropriate for the patient, has always been well tolerated and we did not observe collateral effects.

At the end of 2015, our patient's pool was considerable, and we began to notice that some patient, after the first two weeks on high dose thiamine protocol, would show an initial general improvement followed by a worsening of the original symptoms. We started to think that the cause could be attributed to an excessive dose of thiamine for that specific patient, since we have observed a similar behavior in other diseases [4-6]. Suspending the thiamine protocol for a week led to the regression of the worsening of symptoms. The therapy would then be restarted at halved dosage, which could be further adjusted to reach the correct dosage for the patient and obtain a consistent decrease of the symptoms without any collateral effect. We deem that the appearance of the symptoms of thiamine overdose could have an incidence of about $1 \%$ of the patients treated with $200-300 \mathrm{mg}$ of thiamine per week or with the corresponding oral doses. We also observed a prevalence of thiamine overdose manifestation in newly diagnosed patients, or in patients whose symptoms are mild or who have a small body mass. Over the past months, we began to treat a number of patients of Anglo-Saxon and African origin. Initially we started the therapy with the same schedule used for patients visited in Italy and described in our studies. The vast majority of the patients showed quite soon symptoms attributable to overdose of thiamine, even the same day of the first thiamine administration. Some patients showed, in addition to a general feeling of discomfort, unrest and an overall worsening of the symptoms of Parkinson's disease, also a medium intensity migraine. Such a symptomatology regressed after a few days after the high dose of thiamine was suspended and consequently restarted with halved doses. In other words, these patients have been observed to require, on average, lower doses than those of Italian patients in order to return the same favorable effects on the symptoms. Other ethnicities could be more or less sensitive to the therapy with high dose thiamine. In light of the above, we recommend that colleagues and practitioners begin the treatment with patients of ethnicity other than Italian with doses that are half of what we published previously with reference to the case studies we observed directly. The correct dose, in our experience, is the one that improves the symptoms of Parkinson's disease and improves the sense of balance bringing the score of the pull-test close to normal, as opposed to its altered state due to the disease.

\section{Conflicts of Interests}

On behalf of all authors, the corresponding author states that there is no conflict of interest..

\section{References}

1. Costantini A, Fancellu R (2016) An open-label pilot study with high-dose thiamine in Parkinson's disease. Neural Regen Res 11(3): 406-407.

2. Costantini A, Pala MI, Grossi E, Mondonico S, Cardelli LE, et al. (2015) Long-term treatment with high-dose thiamine in Parkinson disease: An Open-Label Pilot Study. J Altern Complement Med 21(12): 740-747.

3. Costantini A, Pala MI, Compagnoni L, Colangeli M (2013) High-dose thiamine as initial treatment for Parkinson's disease. BMJ Case Rep. pii: bcr2013009289.

4. Costantini A, Pala MI (2013) Thiamine and fatigue in inflammatory bowel diseases: An open-label pilot study. J Altern Complement Med 19(8): 704-708.

5. Costantini A, Tiberi M, Zarletti G, Pala MI, Trevi E (2018) Oral high-dose thiamine improves the symptoms of chronic cluster headache. Case Reports in Neurological Medicine. Article ID 3901619. 
6. Costantini A, Laureti T, Pala MI, Colangeli M, Cavalieri S, et al. (2016)

Long-term treatment with thiamine as possible medical therapy for

Friedreich ataxia. J Neurol 263(11): 2170-2178.

(c) (i) Creative Commons Attribution 4.0 International License

For possible submissions Click Here

Submit Article

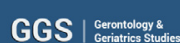

Gerontology \& Geriatrics Studies

Benefits of Publishing with us

- High-level peer review and editorial services

- Freely accessible online immediately upon publication

- Authors retain the copyright to their work

- Licensing it under a Creative Commons license

- Visibility through different online platforms 